\title{
ON THE DIFFERENTIABILITY OF SOLUTIONS OF SYMMETRIC HYPERBOLIC SYSTEMS ${ }^{1}$
}

\author{
GIDEON PEYSER
}

This paper is concerned with the Differentiability Theorem for linear symmetric hyperbolic systems of partial differential equations of the first order. In [1] Friedrichs derives the existence and uniqueness of the strong solution of the Cauchy problem for these systems by using energy inequalities and orthogonal projections. His main tool is the integral mollifier. However, to show that the solution possesses square integrable (strong) derivatives, provided the data determining it is sufficiently smooth, he uses the method of approximations by finite differences. In a different approach, Lax [2] introduces spaces with norms of negative order, such that his solution is already equipped with the necessary square integrable derivatives.

We shall present here a direct proof of the strong differentiability of the solution by applying the existence theorem to the over-determined system resulting from differentiating the given system with respect to all the space variables.

The problem of the strong differentiability of the solution is of particular importance in light of Sobolev's lemma [3], which states that a function possesses continuous derivatives in an appropriate domain, provided it possesses there a sufficient number of square integrable derivatives.

Let $u \equiv\left(u_{1}, \cdots, u_{n}\right)$ denote a vector function of $n$ elements in the $m+1$ independent variables $(t, x) \equiv\left(t, x_{1}, \cdots, x_{m}\right), A_{i}, i=1, \cdots, m$, are symmetric $n \times n$ matrices with sufficiently continuously differentiable elements. $B$ is an $n \times n$ matrix with sufficiently continuously differentiable elements. The domain $R$ is the infinite slab between the two surfaces $S: t=0$ and $T: t=1$.

The given system of equations is

$$
E u \equiv u_{t}+\sum_{i=1}^{m} A_{i} u_{x_{i}}+B u=f
$$

The given data on $S$ is

$$
S u \equiv u(0, x)=\phi(x) .
$$

Received by the editors September 17, 1962.

1 This paper was supported by the National Science Foundation Research Contract NSF-G21328. 
In the following, a vector function will be called smooth if its elements possess a sufficient number of continuous derivatives for the purpose at hand, and have bounded support in the $x$-variables, i.e., vanish for sufficiently large $|x| \equiv\left(x_{1}^{2}+\cdots+x_{m}^{2}\right)^{1 / 2}$.

We say that $u$ has a strong derivative with respect to an independent variable $y$ in the domain $R$, if $u$ has a square integrable $y$ derivative in $R$, i.e., if there exists a sequence of smooth vector functions $u_{k}$ whose $L_{2}$ limit is $u$ and such that the sequence $\left(u_{k}\right)_{y}$ also possesses an $L_{2}$ limit. This $L_{2}$ limit is called the strong derivative of $u$ with respect to $y .^{2}$ We define $u$ as a strong solution of (1) with data (2) if there exists a sequence of smooth vector functions $u_{r}$, such that $u$ is the $L_{2}$ limit of $u_{r}, f$ the $L_{2}$ limit of $E u_{r}$ in $R$, and $\phi$ the $L_{2}$ limit of $S u_{r}$ on $S$.

The Differentiability Theorem. If $f \in L_{2}$ with strong first order $x$-derivatives in $R$, and $\phi \in L_{2}$ with strong first order $x$-derivatives on $S$, then the strong solution $u$ of (1) with data (2), possesses in $R$ strong first order derivatives with respect to all the variables $t$ and $x$.

Note that $f$ need not be strongly differentiable with respect to $t$. We shall derive the strong differentiability of $u$ with respect to the $x$-variables and from (1) then clearly follows the strong differentiability of $u$ with respect to $t$. i.e.,

Replacing $u$ by $e^{-\lambda t} u$ we may assume that $B$ is sufficiently positive,

$$
E u \equiv u_{t}+\sum_{i=1}^{m} A_{i} u_{x_{i}}+(\lambda I+B) u,
$$

where $\lambda$ is a positive constant as large as we please and $I$ is the identity matrix.

$U$ will denote a vector function with $(m+1) n$ elements $U \equiv\left(U_{1}, \cdots, U_{(m+1) n}\right)$. The inner product is defined by

$$
(U, V)=\iint_{R}\left(U_{1} V_{1}+\cdots+U_{(m+1) n} V_{(m+1) n}\right) d t d x
$$

and the norm $\|U\|^{2}=(U, U)$.

$\mathfrak{F C}$ is the Hilbert space obtained by the completion in $R$ of smooth vector functions $U$ under this norm.

$\mathfrak{H C}_{S}$ is the corresponding space on $S$ with inner product

$$
(U, V)_{S}=\int_{S}\left(U_{1} V_{1}+\cdots+U_{(m+1) n} V_{(m+1) n}\right) d x
$$

and norm $\|U\|_{S}^{2}=(U, U)_{S}$.

\footnotetext{
2 For a detailed account of these function spaces, see Lax [2] and Nirenberg [3].
} 
${ }^{3} C_{T}$ is the corresponding space on $T$ with inner product $(U, V)_{T}$ and norm $\|U\|_{r}$.

Let $u \equiv\left(u_{1}, \cdots, u_{n}\right)$ be a vector function with elements in $L_{2}$ and strong $x$-derivatives in $R$. We define $u^{\prime}$ as the vector with $(m+1) n$ elements

$$
u^{\prime} \equiv\left(u_{1}, \cdots, u_{n},\left(u_{1}\right)_{x_{1}}, \cdots,\left(u_{n}\right)_{x_{1}}, \cdots,\left(u_{1}\right)_{x_{m}}, \cdots,\left(u_{n}\right)_{x_{m}}\right),
$$

i.e., $u^{\prime}$ is composed of the elements of $u$ and all their $x$-derivatives. Clearly $u^{\prime} \in \mathcal{F}$. The vectors $u^{\prime}$ form in $R$ a Hilbert space $\mathcal{K}$ which is a closed subspace of $\mathcal{F}$. The vectors $S u^{\prime} \equiv u^{\prime}(0, x)$ resulting from vectors $S u$ with square integrable $x$-derivatives on $S$ form a space $\varkappa_{S}$ which is a closed subspace of $\varkappa_{S}$. Similarly the vectors $T u^{\prime} \equiv u^{\prime}(1, x)$ form a space $\mathcal{K}_{T}$ which is a closed subspace of $\mathcal{H}_{T}$.

We consider the overdetermined system of equations

$$
E u=f
$$

$$
\begin{aligned}
& (\dot{E u})_{x_{1}}=f_{x_{1}} \\
& \dot{(E u})_{x_{m}}=f_{x_{m}},
\end{aligned}
$$

$$
S u=\phi, \quad S u_{x_{1}}=\phi_{x_{1}}, \cdots, S u_{x_{m}}=\phi_{x_{m}} .
$$

Note that we do not differentiate the equation $E u=f$ with respect to $t$.

The system (3)-(4) can be written as

$$
\begin{aligned}
E^{\prime} u^{\prime} & =f^{\prime} \quad\left(\text { this defines the operator } E^{\prime}\right) \\
S u^{\prime} & =\phi^{\prime} .
\end{aligned}
$$

where

$$
\begin{aligned}
& E^{\prime}=\frac{\partial}{\partial t}+\sum_{i=1}^{m} A_{i}^{\prime} \frac{\partial}{\partial x_{i}}+\lambda I^{\prime}+B^{\prime} \\
& A_{i}^{\prime}=\left(\begin{array}{lll}
A_{i} & & 0 \\
& \cdot & \\
& & \\
0 & & A_{i}
\end{array}\right) \quad \text { which is symmetric. }
\end{aligned}
$$

$B^{\prime}$ is an $(m+1) n \times(m+1) n$ matrix and $I^{\prime}$ is the unit matrix.

$u^{\prime}$ is a strong solution of (3)-(4) if there exists a sequence of smooth vector functions $u_{i}^{\prime}$ such that 


$$
\begin{aligned}
\left\|u_{i}^{\prime}-u^{\prime}\right\| & \rightarrow 0 \\
\left\|E^{\prime} u_{i}^{\prime}-f^{\prime}\right\| & \rightarrow 0, \\
\left\|u_{i}^{\prime}-\phi^{\prime}\right\|_{S} & \rightarrow 0, \quad \text { as } i \rightarrow \infty .
\end{aligned}
$$

The following estimates for smooth vector functions $U$, are classical and are deduced in the papers by Friedrichs [1] and by Lax [2].

For any given $k>0$ we can choose $\lambda$ in (5) large enough such that

$$
k\|U\|^{2} \leqq\left(U, E^{\prime} U\right)+\|U\|_{S}^{2}
$$

and if $\left(E^{\prime}\right)^{*}$ denotes the adjoint of $E^{\prime}$, then

$$
k\|U\|^{2} \leqq\left(U,\left(E^{\prime}\right)^{*} U\right)+\|U\|_{T}^{2}
$$

and if $k_{0}=k-1$ then

$$
\begin{aligned}
& k_{0}\|U\|^{2} \leqq\left\|E^{\prime} U\right\|^{2}+\|U\|_{S}^{2}, \\
& k_{0}\|U\|^{2} \leqq\left\|\left(E^{\prime}\right)^{*} U\right\|^{2}+\|U\|_{T .}^{2} .
\end{aligned}
$$

Specifically it follows for smooth $v^{\prime}$ that

$$
\begin{aligned}
& k_{0}\left\|v^{\prime}\right\|^{2} \leqq\left\|E^{\prime} v^{\prime}\right\|^{2}+\left\|v^{\prime}\right\|_{S}^{2}, \\
& k_{0}\left\|v^{\prime}\right\|^{2} \leqq\left\|\left(E^{\prime}\right)^{*} v^{\prime}\right\|^{2}+\left\|v^{\prime}\right\|_{T .}^{2} .
\end{aligned}
$$

From the definition of $E^{\prime}$ in (5) it follows that

$$
E^{\prime} v^{\prime}=(E v)^{\prime} \text {. }
$$

However, $\left(E^{\prime}\right) v^{\prime}$ is not necessarily equal to $\left(E^{*} v\right)^{\prime}$, where $E^{*}$ denotes the adjoint of $E$.

We can write

$$
\left(E^{\prime}\right)^{*} v^{\prime}=\left(E^{*} v\right)^{\prime}+C^{\prime} v^{\prime},
$$

where $C^{\prime}$ is an $(m+1) n \times(m+1) n$ matrix whose elements depend on the elements of $A_{i}$ and $B$ and their derivatives, but are independent of $\lambda$.

The operator $\left(E^{*}\right)^{\prime}$ is defined by the relationship

$$
\left(E^{*}\right)^{\prime} v^{\prime}=\left(E^{*} v\right)^{\prime}
$$

Hence $\left(E^{*}\right)^{\prime} v^{\prime}=\left(E^{\prime}\right) * v^{\prime}-C^{\prime} v^{\prime}$.

The equivalent of (11) for $\left(E^{*}\right)^{\prime}$ is:

$$
k_{0}\left\|v^{\prime}\right\|^{2} \leqq\left\|\left(E^{*}\right)^{\prime} v^{\prime}\right\|^{2}+\left\|v^{\prime}\right\|_{T}^{2} .
$$


There exists a constant $\gamma>0$ such that $\left\|C^{\prime} v^{\prime}\right\|^{2} \leqq \gamma\left\|v^{\prime}\right\|^{2}$. Putting in (15) $k_{0}=4 \gamma$ and using (14) it follows that

$$
4\left\|C^{\prime} v^{\prime}\right\|^{2} \leqq 4 \gamma\left\|v^{\prime}\right\|^{2} \leqq\left\|\left(E^{*} v\right)^{\prime}\right\|^{2}+\left\|v^{\prime}\right\|_{T}^{2} .
$$

We introduce the space $\mathscr{F} \subset \mathcal{K}$. $w^{\prime}$ belongs to $\mathcal{F}$ if there exists a sequence of smooth vector functions $w_{i}^{\prime}$ and vector functions $g^{\prime} \in \Re$, $\psi^{\prime} \in \mathcal{K}_{S}$ such that

$$
\begin{aligned}
\left\|w^{\prime}-w_{i}^{\prime}\right\| & \rightarrow 0, \\
\left\|E^{\prime} w_{i}^{\prime}-g^{\prime}\right\| & \rightarrow 0, \\
\left\|w_{i}^{\prime}-\psi^{\prime}\right\|_{S} & \rightarrow 0, \quad \text { as } i \rightarrow \infty .
\end{aligned}
$$

This extends the definition of the operators $E^{\prime}$ and $S$ to operate on functions $w^{\prime} \in \mathcal{F}$ :

$$
\begin{aligned}
E^{\prime} w^{\prime} & \equiv(E w)^{\prime}=g^{\prime}, \\
S w^{\prime} & =\psi^{\prime} .
\end{aligned}
$$

Inequality (10) continues to hold for functions $w^{\prime} \in \mathcal{F}$.

We introduce the space $\mathfrak{K}^{\times}$which is the product space $\mathfrak{K} \times \mathcal{K}_{S}$ whose elements are all the pairs $\left\{w^{\prime}, \psi^{\prime}\right\} ; w^{\prime} \in \mathcal{K}, \psi^{\prime} \in \mathfrak{K}_{s}$. The inner product in $\mathfrak{K}^{\times}$is defined by

$$
\left(\left\{w_{1}^{\prime}, \psi_{1}^{\prime}\right\},\left\{w_{2}^{\prime}, \psi_{2}^{\prime}\right\}\right)=\left(w_{1}^{\prime}, w_{2}^{\prime}\right)+\left(\psi_{1}^{\prime}, \psi_{2}^{\prime}\right)_{s}
$$

and the norm $\left\|\left\{w^{\prime}, \psi^{\prime}\right\}\right\|^{2}=\left\|w^{\prime}\right\|^{2}+\left\|\psi^{\prime}\right\|_{S}^{2}$.

$\mathfrak{F}^{\times}$denotes the subspace of $\mathfrak{K}^{\times}$whose elements are all the pairs $\left\{(E w)^{\prime}, S w^{\prime}\right\}$ with $w^{\prime} \in \mathcal{F}$. It follows from (10) that $\mathcal{F} \times$ is a closed subspace of $\mathcal{K}^{\times}$. It is our purpose to show that $\mathfrak{F}^{\times}=\mathfrak{K}^{\times}$. This will then complete the proof. We shall use the projection theorem. Suppose that the pair $\left\{h^{\prime}, \sigma^{\prime}\right\} \in \mathcal{K}^{\times}$is orthogonal to the whole space $\mathcal{F}^{\times}$, i.e.,

$$
\left((E w)^{\prime}, h^{\prime}\right)+\left(w^{\prime}, \sigma^{\prime}\right)_{S}=0 \text { for all } w^{\prime} \in \mathcal{F} .
$$

We will then show that $h^{\prime}=0$ and $\sigma^{\prime}=0$ and hence $F^{\times}=\mathfrak{K}^{\times}$. If we restrict $w^{\prime}$ to vector functions in $\mathcal{F}$ with $S w^{\prime}=0$ then $\left((E w)^{\prime}, h^{\prime}\right)=0$. The main problem is then to prove from this that $h^{\prime}=0$. Suppose this is done. We then restrict $w^{\prime}$ to a sequence of smooth functions which approach $\sigma^{\prime}$ on $S$. It follows that $\sigma^{\prime}=0$.

We introduce the mollifiers $J_{\epsilon}$ and $J_{\epsilon}^{*}$. Let $j(t, x)$ be an infinitely differentiable function; $j(t, x) \geqq 0 ; j(t, x)=0$ outside the cube $-1 \leqq t$ $\leqq 1,-1 \leqq x_{i} \leqq 1$ and 


$$
\begin{aligned}
\iint j(t, x) d t d x & =1 \\
J_{\epsilon} q & =\frac{1}{\epsilon^{m+1}} \iint_{R} j\left(\frac{t-\bar{t}+2 \epsilon}{\epsilon}, \frac{x-\bar{x}}{\epsilon}\right) q(\bar{t}, \bar{x}) d \bar{t} d \bar{x}, \\
J_{\epsilon}^{*} q & =\frac{1}{\epsilon^{m+1}} \iint_{R} j\left(\frac{\bar{t}-t+2 \epsilon}{\epsilon}, \frac{\bar{x}-x}{\epsilon}\right) q(\bar{t}, \bar{x}) d \bar{t} d \bar{x}, \\
J_{\epsilon} U & =\left(J_{\epsilon} U_{1}, \cdots, J_{\epsilon} U_{(m+1) n}\right), \\
J_{\epsilon}^{*} U & =\left(J_{\epsilon}^{*} U_{1}, \cdots, J_{\epsilon}^{*} U_{(m+1) n}\right) .
\end{aligned}
$$

If $U \in \mathfrak{F C}$ then, as is well known,

$$
\begin{aligned}
& \left\|J_{\epsilon} U-U\right\| \rightarrow 0 \\
& \left\|J_{\epsilon}^{*} U-U\right\| \rightarrow 0 \quad \text { as } \epsilon \rightarrow 0 .
\end{aligned}
$$

The mollifiers $J_{\epsilon}$ and $J_{\epsilon}^{*}$ commute in the infinite slab $R$ with the $x$-derivatives. Specifically we have for $w^{\prime} \in \mathcal{K}, J_{\epsilon} w^{\prime}=\left(J_{\epsilon} w\right)^{\prime}$ and $J_{\epsilon}^{*} w^{\prime}=\left(J_{\epsilon}^{*} w\right)^{\prime}$.

$J_{\epsilon} w^{\prime}$ and $J_{\epsilon}^{*} w^{\prime}$ are continuously differentiable functions which together with their derivatives are finitely square integrable in $R$. Hence $J_{\epsilon} w^{\prime}$ and $J_{\epsilon}^{*} w^{\prime}$ satisfy (10) and (11). Furthermore $J_{e} w^{\prime}$ and $J_{\epsilon}^{*} w^{\prime}$ vanish in a neighborhood of $T$ and $S$, respectively. Hence we have $\left(\left(E J_{\epsilon}^{*} w\right)^{\prime}, h^{\prime}\right)=0$ for all $w^{\prime} \in \mathcal{K}$. Therefore $\left(\left(E J_{\epsilon}^{*} w\right)^{\prime}, h^{\prime}\right)=\left(E^{\prime}\left(J_{\epsilon}^{*} w\right)^{\prime}, h^{\prime}\right)$ $=\left(E^{\prime} J_{e}^{*} w^{\prime}, h^{\prime}\right)=\left(w^{\prime},\left(E^{\prime} J_{c}^{*}\right)^{*} h^{\prime}\right)=0$, where $\left(E^{\prime} J_{c}^{*}\right)^{*}$ is the adjoint integral operator of $E^{\prime} J_{\epsilon}^{*}$. From Friedrichs' lemma [2, Lemma 14], it follows that

Hence

$$
\left\|\left(E^{\prime} J_{\epsilon}^{*}\right)^{*} U-\left(E^{\prime}\right)^{*} J_{\epsilon} U\right\|^{2} \rightarrow 0 \quad \text { as } \epsilon \rightarrow 0 .
$$

$$
\left(w^{\prime},\left(E^{\prime}\right) * J_{\epsilon} h^{\prime}\right) \rightarrow 0 \quad \text { for all } w^{\prime} \in \mathcal{K} .
$$

Since $h^{\prime}$ is fixed we can consider (17) as a sequence of linear bounded functionals of $w^{\prime}$. This sequence converges for all $w^{\prime} \in \mathcal{K}$. Therefore the norms of these functionals are uniformly bounded by a constant $M$. Hence, choosing $w^{\prime}=\left(E^{*} J_{\epsilon} h\right)^{\prime}$, we have:

$$
\begin{aligned}
M\left\|\left(E^{*} J_{\epsilon} h\right)^{\prime}\right\| & \geqq\left(\left(E^{*} J_{\epsilon} h\right)^{\prime},\left(E^{\prime}\right)^{*} J_{\epsilon} h^{\prime}\right)=\left\|\left(E^{*} J_{\epsilon} h\right)^{\prime}\right\|^{2}+\left(\left(E^{*} J_{\epsilon} h\right)^{\prime}, C^{\prime} J_{\epsilon} h^{\prime}\right) \\
& \geqq \frac{1}{2}\left\|\left(E^{*} J_{\epsilon} h\right)^{\prime}\right\|^{2} .
\end{aligned}
$$

(The last inequality follows from (16).) Hence

$$
\left\|\left(E^{*} J_{\epsilon} h\right)^{\prime}\right\| \leqq 2 M \text {. }
$$

Therefore $\left(E^{*} J_{\epsilon} h\right)^{\prime}$ is a sequence of functions in $\mathscr{K}$ with uniformly 
bounded norms. Now, from a sequence of functions with uniformly bounded norms we can choose a subsequence which converges weakly. From the Banach-Sachs theorem, see Riesz-Nagy [4, §38], it follows that from this weakly convergent sequence we can select a subsequence whose arithmetic means converge strongly. Since $\left(E^{*}\right)^{\prime}$ is a linear operator, we have a sequence of continuously differentiable vector functions $v_{i}^{\prime}$ in $\mathcal{K}$ which are the arithmetic means of a subsequence of $J_{\epsilon} h^{\prime}$, such that $\left(E^{*}\right)^{\prime} v_{i}^{\prime}\left(=\left(E^{*} v_{i}\right)^{\prime}\right)$ converges strongly. Since $J_{t} h^{\prime}$ vanishes on $T$ we have that $\left\|v_{i}^{\prime}\right\|_{T}=0$. Furthermore

$$
\left\|v_{i}^{\prime}-h^{\prime}\right\| \rightarrow 0 \text {. }
$$

We denote the strong limit of $\left(E^{*} v_{i}\right)^{\prime}$ by $e^{\prime}$. Substituting $e^{\prime}$ for $w^{\prime}$ in (17) we deduce that $\left(e^{\prime},\left(E^{\prime}\right) *^{\prime} v_{i}^{\prime}\right) \rightarrow 0$. Furthermore

$$
\begin{aligned}
\left(e^{\prime},\left(E^{\prime}\right)^{*} v_{i}^{\prime}\right)= & \left(e^{\prime},\left(E^{*} v_{i}\right)^{\prime}\right)+\left(e^{\prime}, C^{\prime} v_{i}^{\prime}\right) \geqq\left(e^{\prime},\left(E^{*} v_{i}\right)^{\prime}\right)-\frac{1}{2}\left\|e^{\prime}\right\|\left\|\left(E^{*} v_{i}\right)^{\prime}\right\| \\
& \left.\rightarrow \frac{1}{2}\left\|e^{\prime}\right\|\right|^{2} .
\end{aligned}
$$

Hence $e^{\prime}=0$, i.e., $\left\|\left(E^{*} v_{i}\right)^{\prime}\right\| \rightarrow 0$. (15) implies that $\left\|v_{i}^{\prime}\right\| \rightarrow 0$. From (19) it now follows that $h^{\prime}=0$, which concludes the proof.

By repeated application of the Differentiability Theorem we deduce the higher order differentiability of the solution of (1)-(2), provided $f$ and $\phi$ possess higher order derivatives.

Higher Order Differentiability Theorem. If $r$ and $s$ are nonnegative integers with $s \leqq r$ and if $f$ possesses in $R$ all the strong derivatives of the form $D_{t}^{\alpha} D_{x_{1}}^{\beta_{1}} \cdots D_{x_{m}}^{\beta_{m}}, \alpha \leqq s, \alpha+\beta_{1}+\cdots+\beta_{m} \leqq r$, and if $\phi$ possesses on $S$ all the strong derivatives of the form $D_{x_{1}}^{\beta_{1}} \cdots D_{x_{m}}^{\beta_{m}}$, then the solution $u$ of (1)-(2) possesses in $R$ all the strong derivatives of the form

$$
D_{t}^{\alpha^{\prime}} D_{x_{1}}^{\beta_{1}} \cdots D_{x_{m}}^{\beta_{m}}, \quad \alpha^{\prime} \leqq s+1, \quad \alpha^{\prime}+\beta_{1}+\cdots+\beta_{m} \leqq r .
$$

\section{BiBLIOGRAPHY}

1. K. O. Friedrichs, Symmetric hyperbolic linear differential equations, Comm. Pure Appl. Math. 7 (1954), 345-392.

2. P. D. Lax, On Cauchy's problem for hyperbolnc equations and the differentiability of solutions of elliptic equations, Comm. Pure Appl. Math. 8 (1955), 615-633.

3. L. Nirenberg, Remarks on strongly elliptic partial differential equations, Comm. Pure Appl. Math. 8 (1955), 649-676.

4. F. Riesz and B. Sz.-Nagy, Functional analysis, Ungar, New York, 1955.

Pratt Institute 